\title{
Descripción de los ingresos y reingresos de recién nacidos prematuros en España:
}

\author{
causas y costes
}

Description of preterm neonates admitted and readmitted in Spain: causes and costs

Descrição de internação e reinternação de recémnascidos prematuros na Espanha: causas e custos

\author{
Juliana Jesus Alves', María D. Redín Areta², Blanca Marín
}

Fernández ${ }^{2}$

\begin{abstract}
${ }^{1}$ Licenciada en Enfermería por la Universidade de São Paulo (USP) campus Ribeirão Preto (Brasil), enfermera en el Hospital Santa Luzia red D'Or en Brasília (Brasil) y en el Instituto de Cardiologia do Distrito Federal en Brasília (Brasil). Máster en Gestión de Cuidados en Enfermería por la Universidad Pública de Navarra (España).Correo electrónico: julianajalves@hotmail.com

2Profesoras Doctoras Titulares (TU) del área de Enfermería en la Universidad Pública de Navarra (España). Correos electrónicos: dolores.redin@unavarra.es, blanca.marin@unavarra.es
\end{abstract}

Cómo citar este artículo en edición digital: Alves, J.J., Areta, D.R. \& Marín Fernández, B. (2019).

Descripción de los ingresos y reingresos de recién nacidos prematuros en España: causas y costes. Cultura de los Cuidados (Edición digital), 23(53). Recuperado de http://dx.doi.org/10.14198/cuid.2019.53.28

Correspondencia: Juliana de Jesus Alves.QRSW 6 bloco B4 apto 103, sudoeste, Brasília-DF, Brasil. Cep: 70675-524

Correo electrónico de contacto: julianajalves@hotmail.com Recibido: 27/09/2018; Aceptado: 15/01/2019

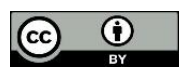

\section{ABSTRACT}

Objective: Identify the primary cause of the hospital admission and readmission of premature infants and evaluate the costs of the readmission between the years 2000 and 2011 in the Comunidade Foral de Navarra hospital in Pamplona, Spain.

Methods: This is a descriptive, retrospective study involving analysis of 297 premature infants who were admitted to the neonatal Intensive Care Unit and were subsequently readmitted at another division of the aforementioned hospital, at least once, during the sixty days after discharge.

Results: The majority of the cases studied $(91.25 \%)$ were re-hospitalized only once, with an average stay of 4.61 days. Bronchiolitis and asthma were the most common cause for patient readmission.

Conclusion: The author highlights the importance of parental presence during the child's hospitalization, as they can provide detailed information on the normal characteristics of their child and the potential health issues associated with prematurity. If the child presents any symptoms, the parents will know to seek medical help in the early stages, reducing the risk of complications and future hospitalizations.

Keywords: Premature, readmitted, costs. 


\section{RESUMO}

Objetivo: Identificar as principais causas de internação e reinternação hospitalar de recém-nascidos prematuros e avaliar os custos que estas reinternações geraram entre os anos de 2000 a 2011 no Hospital Virgen Del Camino (Pamplona), Espanha.

Metodologia: Estudo descritivo, retrospectivo, com análise de 297 bebes prematuros que estiveram internados na UTI neonatal e reinternaram em algum setor do hospital em questão pelo menos uma vez, em até 60 dias após sua alta.

Resultados: A maior parte dos casos (91, $25 \%$ ) apresentaram uma única reinternação. O tempo médio de estadia na reinternação foi de 4, 61 dias. As principais causas que levaram a uma reinternação foram bronquites e asma sem complicações.

Conclusão: Fazer com que os país permanesçam com seus filos em sua hospitalização podem proporciona-los maior conhecimento sobre o padrão de normalidade de seus filos, conhecer as principais doenças e os principais problemas asociados a prematuridade, fazendo com que os país procurem ajuda médica na fase inicial da doença, podemdo assim diminuir as chances de uma reinternação hospitalar.

Palavras chave: Prematuro, reinternação, custos.

\section{RESUMEN}

Objetivo: Identificar las principales causas de ingresos y reingresos hospitalarios de recién nascidos prematuros y evaluar los costes que los reingresos generan entre los años 2000 y 2011 en el Hospital Virgen Del Camino (Pamplona), España.

Métodos: Estudio descriptivo, retrospectivo, con análisis de 297 niños prematuros que estuvieron ingresados en la
UCI neonatal y reingresaron en algún servicio del hospital en cuestión, al menos una vez, en los 60 días tras el alta hospitalaria.

Resultados: La mayor parte de los casos $(91,25 \%)$ presentaron un único reingreso. La estancia media de reingreso fue de 4,61 días. Las bronquitis y asma sin complicaciones constituyen la causa más común de reingreso.

Conclusión: Intentar que los padres permanezcan con sus hijos cuando éstos están hospitalizados les puede proporcionar una mayor formación acerca de las características normales de su hijo, de las enfermedades y problemas asociados a la prematuridad, haciendo con que los padres busquen ayuda médica en la inicial de la enfermedad pudiendo disminuir el riesgo de un posible reingreso hospitalario.

Palabras clave: Prematuro; reingresos; costes.

\section{INTRODUCCIÓN}

La Organización Mundial de la Salud (OMS) definió como prematuro a todo aquel recién nacido con menos de 37 semanas completas de gestación (259 días) mientras que el recién nacido a término nace entre las semanas 37 y la 42. Se estima que hoy en día nacen, en todo el mundo, 15 millones de niños antes de tiempo al año y que, de estos, cerca de 1 millón mueren por complicaciones de la prematuridad. Además, muchos de los que sobreviven sufren problemas visuales, auditivos, motores y cognitivos a lo largo de la vida (Howson, Kinney e Lawn, 2012).

La magnitud del problema, así como las disparidades entre países, nos apuntan que de los 11 países con índices de nacimientos prematuros superiores al 15 por ciento, 
todos menos dos (Indonesia y Pakistán) están en África subsahariana. Los nacimientos prematuros representan el $11.1 \%$ de los nacimientos vivos del mundo, $60 \%$ de ellos en Asia meridional y África subsahariana. En los países más pobres, en promedio, $12 \%$ de los bebés nacen demasiado pronto, en comparación con el 9\% en los países de mayores ingresos (Howson, Kinney e Lawn, 2012).

Sin embargo, el problema de los nacimientos prematuros no se limita a los países de bajos ingresos. Estados Unidos y Brasil también tienen un elevado número de nacimientos prematuros. Por ejemplo, en los Estados Unidos, alrededor del $12 \%$, o más de uno de cada nueve nacimientos, son prematuros. En España este valor es de 7, 4\% (Howson, Kinney e Lawn, 2012).

El nacimiento prematuro se da por diversas causas siendo las más comunes embarazos múltiples, embarazos adolescentes, lapsos cortos de tiempo entre nacimientos, peso inadecuado de la madre antes del embarazo (bajo peso $u$ obesidad), abuso de substancias como el tabaco y alcohol e infecciones y enfermedades crónicas, como la diabetes y la hipertensión. Sin embargo, frecuentemente no se identifica una causa pudiendo ser genética (Howson, Kinney e Lawn, 2012).

La sobrevida de los prematuros aumentó significativamente en las últimas décadas debido principalmente a los avances en la medicina perinatal, la llegada de las unidades de cuidados intensivos neonatales, la introducción y el desarrollo de la ventilación mecánica, la incorporación de técnicas de monitorización no invasiva $y$, principalmente, por el uso de corticoides prenatales para acelerar la maduración pulmonar y la introducción del surfactante exógeno (Ramírez e Hübner, 2002).

La disponibilidad de los recursos necesarios en la atención perinatal justifica que, en países con altos ingresos, la mitad de los bebés nacidos a las 24 semanas de gestación sobrevivan, mientras que, en países de bajos ingresos, la mitad de los bebés nacidos a las 32 semanas de gestación siguen muriendo debido a la falta de atención (Howson, Kinney e Lawn, 2012).

El recién nacido pre termino (RNPT), por el grado de inmadurez de sus órganos y sistema inmunológico tendrá una mayor predisposición, comparado al recién nacido a término (RNAT), a padecer problemas respiratorios, cardíacos, visuales, auditivos y neurológicos además de problemas secundarios relacionados con las largas estancias en la UCI.

La evolución de los niños con alteraciones en su desarrollo dependerá mucho de la fecha de la detección y del momento del inicio de la Atención Temprana. Cuanto menor sea el tiempo de privación de los estímulos, menor será el retraso. En este proceso es indispensable la implicación familiar en el rol cuidador para favorecer la interacción afectiva y emocional así como para la eficacia de los tratamientos.

Según Donabedian (1986), el propósito de los sistemas de atención a la salud es proporcionar la mayor calidad al menor costo, de la manera más equitativa y al mayor número de personas.

Frente al volumen de recursos implicados el conocimiento de la producción de los servicios sanitarios es escaso y el nivel de información no permite conocer adecuadamente cuáles son los problemas de salud más frecuentes atendidos, cómo son tratados, con qué costo y sobre todo con qué resultados.

Como consecuencia, durante los últimos años se han realizado numerosos esfuerzos para aumentar la recolección y estandarización de datos y también están 
siendo desarrollados proyectos de investigación destinados a obtener mejoras en el conocimiento del funcionamiento $y$ eficacia de los servicios sanitarios.

En el ámbito hospitalario la actividad se ha medido durante mucho tiempo a través de indicadores cuantitativos, como la estancia o el número de consultas sin considerar ningún componente clínico (Paolillo et al., 2008)

A finales de la década de 1970 se pusieron a disposición de los hospitales herramientas de medición del producto hospitalario basadas en parámetros clínicos. Para ello, se desarrolló el concepto de casuística hospitalaria que representa las respuestas del hospital a las diversas tipologías de pacientes atendidos. Si se pudiesen medir los diversos tipos de pacientes, se podrían prever las necesidades asistenciales de un servicio o de todo un hospital (Oliván, 1997).

La casuística hospitalaria puede ser medida de diferentes formas: mediante índices, como la gravedad (sistemas de isogravedad), así ocurre con el sistema APACHE, o mediante clasificaciones de pacientes que miden el consumo de recursos (grupos de iso-consumo) como ocurre con los Grupos Relacionados por el Diagnóstico (GRD) (Paolillo, E., et al.; Oliván, 1997).

Los avances en el tratamiento de los prematuros en Unidades de Cuidados Intensivos Neonatales han ayudado a mejorar sensiblemente su supervivencia. Sin embargo, estos niños siguen siendo vulnerables a muchas complicaciones, incluyendo problemas respiratorios, gastrointestinales, del sistema inmune, sistema nervioso central, de la audición y de la visión. Problemas a más largo plazo pueden incluir parálisis cerebral, retraso mental, deterioro visual y de audición, aspectos de comportamiento y socioemocionales, dificultades de aprendizaje y menor salud y crecimiento.

En general, mientras más inmaduro sea el prematuro, mayor es el grado del soporte de vida necesario y más larga es la estancia requerida en una Unidad de Cuidados Intensivos Neonatales, la estancia hospitalaria en total tiende a ser más larga, mientras que los riesgos de reingresos aumentan considerablemente. Las consecuencias para los niños prematuros a lo largo de la vida pueden ser significativas y a menudo se requiere un amplio rango de servicios y de apoyo social.

En Estados Unidos el Comité sobre Análisis de Parto Prematuro y Garantía de Resultados Saludables estima que la carga económica anual para la sociedad, asociada con nacimiento prematuro en Estados Unidos, fue, como mínimo, de 26.2 billones de dólares en 2005 o 51, 600 por niño nacido prematuro. Cerca de dos tercios de este importe se produjo por el cuidado médico (Behrman e Butler, 2007)

Dos estudios recientes demostraron que el coste de la atención para los bebés prematuros después del alta hospitalaria inicial es mayor que el de recién nacidos a término. Uno analizaba el costo médico total en los primeros dos años de vida de 71 niños con peso extremadamente bajo al nacer (recién nacidos peso <1000 g) nacidos en Finlandia desde 1996 hasta 1997 (Tommiska, Tuomnien e Fellman, 2003). En el otro estudio se examinaron los costes y las causas de hospitalización en los primeros 5 años de vida de 240.000 niños de todas las edades gestacionales nacidos en el Reino Unido entre 1970 y 1993 (Petrou et al., 2003).

Estos autores encontraron que el costo total medio de los bebés de bajo peso extremo al nacer que llegan a sobrevivir fue de 105.000 euros. El gasto de la estancia hospitalaria inicial supuso el $64 \%$ del valor total. También demostró que el peso al nacer 
se correlacionó negativamente con el costo inicial (los bebés más prematuros, <28 semanas de gestación, tienen un costo de 30 veces más que los recién nacidos a término), pero no con el coste del alta inicial.

De acuerdo con la literatura revisada y la importancia del tema en el contexto de la gestión hospitalaria y con el objetivo de buscar nuevas perspectivas de investigación en el área de Gestión de Servicios Neonatales, se plantea en el presente estudio evaluar los costes que generan estos ingresos y reingresos.

Por lo tanto los principales objetivos del estudio son identificar las principales causas de ingresos y reingresos hospitalarios $y$ evaluar los costes que los reingresos generan.

\section{METODOLOGÍA}

Se basa en un estudio descriptivo, retrospectivo, observacional, cuyo ámbito de estudio fue la base de datos del Conjunto Mínimo Básico de Datos (CMBD) del Hospital Virgen del Camino, Pamplona, Navarra, España. El estudio buscó evaluar, en el periodo de enero del 2000 a diciembre de 2011, todas las causas de los ingresos y reingresos hospitalarios de recién nacidos prematuros nascidos en este hospital.

Participaron en el estudio 297 niños prematuros que estuvieron ingresados en la UCI neonatal del Complejo Hospitalario B y reingresaron en algún servicio hospitalario del hospital en cuestión, al menos una vez, en los 60 días tras el alta hospitalaria. Fueron excluidos del estudio aquellos que tenían como diagnóstico principal o secundario más de 37 semanas de gestación y 3 casos en los que no se encontró la edad gestacional y, por tanto, no se pudo saber si eran prematuros.

Fue utilizado para calcular los costos y analizar todos los ingresos y reingresos la última versión del Manual de Descripción de los Grupos Relacionados por el Diagnóstico (AP-GRD v.25.0, 2010)

Los GRDs de ingreso en la UCI neonatal y los GRDs de reingresos fueron agrupados por semejanza/año.

Para calcular los costes fue multiplicado el peso de cada GRD por el precio de unidad de GRD de cada año, estipulado por el Servicio Navarro de Salud y con valores reajustados en los años de 1999, 2002, 2006 y 2010, publicados en los Boletines Oficiales de Navarra. Los años 2000 y 2001 se obtuvieron con valor en pesetas y fueron pasados a euros. Después de calculado el valor de cada GRD, fueron agrupados por año para obtener los gastos anuales tanto en los ingresos como en los reingresos.

Todos los aspectos éticos fueron seguidos con rigoroso criterio.

\section{RESULTADOS}

En el periodo estudiado el número de recién nacidos prematuros atendidos en el Hospital Virgen del Camino fue de 2.429.

El 12, 22\% de estos bebés (297) tuvieron que ser ingresados al menos una vez, en un periodo de 60 días tras alta hospitalaria.

Descripción del ingreso tras el parto. Ingresos:

Un total de 297 niños prematuros precisaron un nuevo ingreso en el periodo de tiempo estudiado. De ellos, el 61, 27\% fueron niños y el 38, 72\% niñas.

La estancia media de ingreso en el momento del nacimiento fue de 32, 36 días con variación de 0 hasta 267 días.

Las causas de ingreso tuvieron un total de 24 GRDs distintos, con una variación del número de casos en cada uno que osciló entre 55 y 1. 
Las causas más comunes de admisión en la UCI neonatal fueron:

- En primer lugar, con 55 casos, neonato con peso al nacer 1000 a $1499 \mathrm{~g}$ sin procedimiento quirúrgico significativo, alta con vida (GRD 607).

- Seguido de 36 casos, con neonato peso al nacer 2000 a $2499 \mathrm{~g}$ sin procedimiento quirúrgico significativo, diagnóstico neonato normal (GRD 620).

- En tercer lugar, con 29 casos, neonato peso al nacer 1500 a $1999 \mathrm{~g}$ sin procedimiento quirúrgico significativo sin múltiples problemas mayores (GRD 612).

- Con 26 casos, en cuarto lugar, neonatos peso al nacer 1500 a $1999 \mathrm{~g}$ sin procedimiento quirúrgico significativo con otros problemas (GRD 614).

- En quinto lugar, con 23 casos neonato peso al nacer 2000 a $2499 \mathrm{~g}$ sin procedimiento quirúrgico significativo con problemas mayores (GRD 618).

- Seguido de 19 casos peso al nacer menor de $750 \mathrm{~g}$ alta con vida (GRD 604).

- En séptimo lugar, con 16 casos de neonato peso al nacer 1500 a $1999 \mathrm{~g}$ sin procedimiento quirúrgico significativo con múltiples problemas mayores o ventilación mecánica más de 95 horas (GRD 611).

- Seguido de 11 casos, neonato peso al nascer mayor de $2499 \mathrm{~g}$ sin procedimiento quirúrgico significativo con diagnóstico neonato normal (GRD 629).

- En noveno lugar, con 10 casos, neonato peso al nacer 1500 a 1999, g con procedimiento quirúrgico significativo con múltiples problemas mayores (GRD 609) y, con 9 casos, neonato peso al nacer mayor de $2499 \mathrm{~g}$ sin procedimiento quirúrgico significativo con problema mayor (GRD 627).
Podemos destacar que 47 de estos casos corresponden a niños que presentan un diagnóstico de neonato normal (GRDs 620 y 629). La estancia media de estos niños fue de 8,48 días con variación de 0 hasta 25 días.

Hay, por otro lado, 45 bebés de éstos que son de alto riesgo, o sea, presentaron uno de los factores a seguir: múltiples problemas mayores, ventilación mecánica o extremo bajo peso (GRDs 604, 609 y 611). La estancia media para estos casos fue de 57, 86 días con variación de 19 hasta 133 días.

Descripción de los ingresos tras el alta inicial. Reingresos:

La mayor parte de los casos, 271 (91, 25\%), presentaron un único reingreso.

En este grupo encontramos que el 60, 33\% eran niños y $39,67 \%$ niñas.

La estancia media de reingreso fue de 4, 61 días con variación de 0 hasta 57 días.

Los problemas que ocasionaran los reingresos tuvieron un total de 79 GRDs distintos.

El gráfico II recoge la información correspondiente a los GRD que presentaron 3 casos o más. Las bronquitis y asma sin complicaciones constituyen la causa más común de reingreso con un total de 34 casos, en segundo lugar encontramos los Procedimientos sobre hernia con 30 casos, siendo las Hemorragias con complicaciones, con 19 casos, la tercera causa en orden de frecuencia, seguida de bronquitis y asma con complicación mayor con 14 casos. También, con 14 casos, está otitis media e infecciones del tracto respiratorio superior. Con 10 casos está signos y síntomas respiratorios sin complicaciones.

Fuente: Unidad de Codificación del complejo hospitalario B, Pamplona, Comunidad Foral de Navarra. Elaboración propia. 


\section{Cultura de los Cuidados}

Como es lógico, ante un número tan elevado de GRDs distintos, el número de casos correspondientes a alguno de ellos fue muy pequeño. En este sentido, observamos que más de la mitad de los GRDs (58\%) tuvieron apenas un caso, con dos casos se presentó el $14 \%$.

Cabe destacar que los problemas respiratorios, en conjunto, fueron los responsables del mayor número de reingresos alcanzando un total de 114 casos. En segundo lugar, encontramos los problemas digestivos con 88 casos, seguidos, ya con menor incidencia, de problemas inmunológicos (13 casos); endocrinos y metabólicos, con 10 casos; urinarios, con 5 casos; y circulatorios, con 3 casos.

\section{TABLA I: Principales Sistemas}

\section{N. ${ }^{\circ}$ de casos}

Sistema Respiratorio

Sistema Digestivo

Sistema Inmunológico

Sistema Endocrino, nutrición y metabolismo

Sistema Urinario

Sistema Circulatorio
114

88

13

10

5

3

FUENTE: Unidad de Codificación del complejo hospitalario B, Pamplona, Comunidad Foral de Navarra. Elaboración propia

La edad de reingreso ocurrió en mayor porcentaje (39\%) entre 31 y 60 días después de la alta hospitalaria; en segundo lugar, encontramos los ingresos producidos de 61 a 90 días tras el alta (27\% ) y, en tercer lugar, se producen los reingresos de los recién nacidos hasta 30 días (18\%) después del alta.

Los principales servicios de alta en las readmisiones hospitalarias fueron pediatría con 226 casos; cirugía infantil, con 53 casos, seguida de neonatología, con 15 casos.

Como se muestra en la tabla II, la estancia media más prolongada en el reingreso corresponde a problemas del sistema musculo esquelético y tejido conjuntivo con estancia media de 10, 5 días; seguida de las enfermedades y trastornos del riñón y vías urinarias, con 9, 12 días de ingreso; $y$, en tercer lugar, de las enfermedades del sistema endocrino, con 7, 5 días. Los problemas del aparato respiratorio se sitúan en sexto lugar con una estancia media de 5, 66 días.

\section{Casos con más de un reingreso:}

Hubo un total de 26 bebés que reingresaron más de una vez. De estos niños, 22 precisaron dos reingresos, en 3 casos fueron necesarios tres ingresos sucesivos $y$, finalmente, un niño reingresó cuatro veces.

En este grupo el 76, 9\% (20 bebés) eran niños y $23,1 \%$ ( 6 bebés) niñas.

En relación a la estancia media observamos que en el ingreso fue de 44, 5 días mientras en los reingresos fue de 5, 2 días. 


\section{Cultura de los Cuidados}

\begin{tabular}{|l|c|}
\hline TABLA II: Principales causas. Reingresos por sistemas & Estancia media \\
\hline Sistema nervioso & 6,16 \\
\hline Enfermedades y trastornos de ojo & 2,33 \\
\hline Enfermedades y trastornos del oído, nariz y faringe & 1,83 \\
\hline Enfermedades y trastornos del aparato respiratorio & 5,66 \\
\hline Enfermedades y trastornos del aparato circulatorio & 6,71 \\
\hline Enfermedades y trastornos del aparato digestivo & 4,24 \\
\hline Sistema muscoloesquelético y tejido conjuntivo & 10,5 \\
\hline Enfermedades y trastornos de piel, tejido subcutáneo o mama & 1 \\
\hline Enfermedades del sistema endocrino, nutrición y metabolismo & 7,5 \\
\hline Enfermedades y trastornos del riñón y vías urinarias & 9,12 \\
\hline Sangre, órganos hematopoyéticos y sistema inmunológico & 0 \\
\hline Enfermedades infecciosas & 3,36 \\
\hline Trastornos mentales & 2 \\
\hline
\end{tabular}

FUENTE: Unidad de Codificación del complejo hospitalario B, Pamplona, Comunidad Foral de Navarra. Elaboración propia

Hubo un total de 30 GRDs distintos siendo el responsable del mayor número de ingresos la bronquitis y asma sin complicaciones, con 11 casos.

Dos de los bebés que reingresan dos veces tienen el diagnóstico de alta, en el ingreso, de peso al nacer menor de $750 \mathrm{~g}$ y reingresan por edema pulmonar e insuficiencia respiratoria y bronquitis y asma.

De los bebés que tuvieron 3 reingresos, uno tenía el diagnóstico inicial de neonato con peso al nacer 2000-2499g, con procedimiento quirúrgico significativo, sin múltiples problemas mayores (GRD 616), volviendo a reingresar dos veces por trastornos nutricionales y metabólicos misceláneos y otra por procedimiento mayor de estómago, esófago, duodeno, intestino delgado y grueso con complicaciones mayores. Los otros dos niños presentaron el diagnóstico de neonato con peso al nacer 1000-1499g, sin procedimiento quirúrgico significativo, alta con vida. Uno de ellos volvió a reingresar por bronquitis y asma y otros diagnósticos del aparato respiratorio. El otro, por trastornos degenerativos del sistema nervioso, septicemia con complicación mayor y craneotomía.

El bebé que reingresa en cuatro ocasiones tiene el diagnóstico inicial de neonato con peso al nacer $1500-1999 \mathrm{~g}$ con procedimiento quirúrgico significativo, con múltiples problemas mayores. Reingresa por procedimientos sobre riñón, uréter y vejiga; infecciones de riñón y tracto urinario y obstrucción congénita de la unión ureterovesical.

Los años de 2007 y 2011 tuvieron los costes más altos mientras los años de 2005 y 2010 tuvieron menos gastos.

En la tabla III podemos notar que los costes de los ingresos son mayores que los reingresos. 


\begin{tabular}{|r|r|r|r|r|r|}
\hline $\begin{array}{c}\text { TABLA III: } \\
\text { Costes Anuales } \\
\text { (Euros) }\end{array}$ & Ingresos & & Reingresos & & \\
\hline & Coste Medio & Coste Total & Coste Medio & Coste Total & TOTAL \\
\hline 2000 & 17066,60 & 392531,89 & 1146,69 & 40134,06 & 432665,95 \\
\hline 2001 & 14601,77 & 350442,66 & 1380,61 & 49702,07 & 400144,73 \\
\hline 2002 & 40368,71 & 363318,46 & 3556,63 & 56906,15 & 420224,61 \\
\hline 2004 & 34211,88 & 376330,71 & 3358,67 & 53738,76 & 430069,47 \\
\hline 2005 & 22188,45 & 399392,22 & 3635,70 & 69078,40 & 468470,62 \\
\hline 2006 & 29919,85 & 269278,68 & 2298,15 & 32174,20 & 301452,88 \\
\hline 2007 & 53751,79 & 371269,76 & 3026,57 & 48425,22 & 419694,98 \\
\hline 2009 & 47630,95 & 804464,31 & 3473,43 & 59048,43 & 863512,74 \\
\hline 2010 & 23546,80 & 282561,61 & 4262,44 & 59674,28 & 342235,89 \\
\hline 2011 & 27342,57 & 765592,20 & 3531,51 & 98882,52 & 864474,72 \\
\hline
\end{tabular}

FUENTE: Unidad de Codificación del complejo hospitalario B, Pamplona, Comunidad Foral de Navarra. Elaboración propia

\section{DISCUSIÓN Y CONCLUSIÓN}

\section{Ingresos:}

Según los datos encontrados en el presente estudio, las principales causas de ingreso en la UCI neonatal fueron bajo peso al nacer asociado a prematuridad. En los partos prematuros sobretodo antes de la 32.. semana de gestación, existen mayores riesgos de complicación para el recién nacido, como problemas respiratorios por inmadurez pulmonar, riesgo de hemorragia interventricular por fragilidad de los capilares cerebrales y susceptibilidad a las infecciones por la inmadurez del sistema inmunológico (Tamez e Silva, 2002). Todos estos factores explican el hecho de que cuanto más prematuro sea el recién nacido, menor su peso, mayor será su estancia en la UCI neonatal y mayor riesgo de complicaciones.

La estancia media de ingreso fue de 32,36 días, mientras que la estancia media de reingreso fue de 4, 61 días. Podemos sugerir que debido la inmadurez de los órganos y los múltiples problemas que genera la prematuridad, la estancia en el ingreso es mayor que en el reingreso, ya que en este último los bebés vuelven por una causa específica.

El número de GRDs también varía en los dos grupos, mientras en el ingreso se agrupan en 24 GRDs distintos, en los reingresos alcanzan los 79 .

\section{Reingresos:}

Nuestros resultados coinciden con los de Underwood, Danielsen e Gilbert, 2007 y Hayakawa at al, 2010, que encontraron como principales causas de reingresos hospitalarios las de origen respiratorio incluyendo bronquitis, asma, bronquiolitis y neumonías. 
El desarrollo del pulmón fetal así como la producción de surfactante son necesarios para la función respiratoria normal. La síntesis de surfactante se inicia a partir de la 23. a semana de gestación. La maduración anatómica y funcional de los pulmones requiere por lo menos 35 semanas de gestación. En los recién nacidos prematuros estas funciones están comprometidas, lo que causa una mayor incidencia de problemas respiratorios (Petrou at al., 2003; Bonitto 2012).

Las infecciones causadas por virus son más frecuentes en climas templados, durante los meses de invierno y primavera, cuando las temperaturas son más bajas y hay la presencia de partículas en el aire. En España destacan, entre otros, el virus respiratorio sincital, influenza, parainfluenza, enterovirus, rinovirus $\mathrm{y}$ coronavirus. ${ }^{15}$ Podemos entender que, como en la región de Pamplona las temperaturas son extremas, o sea, en verano, muy calurosas; y en invierno, muy frías, además de ser una ciudad muy arbolada y de la presencia de partículas suspendidas en el aire en primavera, se favorece la aparición de enfermedades respiratorias).

La observación de los padres, principalmente en invierno y en primavera, así como el conocimiento de los principales signos y síntomas son valiosos para establecer un diagnóstico temprano y acertado. Sus percepciones respecto al curso de la enfermedad y su inicio son tan importantes como el conocimiento de la fisiopatología de cada enfermedad común a los prematuros o posible de desarrollar por su hijo, sea por la prematuridad o por histórico de tal enfermedad en el ámbito familiar.

En nuestro estudio, la mayoría de los reingresos se producen pasados 30 días tras el alta hospitalaria.
En este sentido, cabe plantearnos si el intentar que los padres permanezcan con sus hijos cuando éstos están hospitalizados les puede proporcionar una mayor formación acerca de las características normales de su hijo, de las enfermedades y problemas asociados a la prematuridad y de sus complicaciones para que, si el bebé desarrolla alguna enfermedad, los padres busquen ayuda médica cuando la misma aún este en su fase inicial, pudiendo disminuir el riesgo de un posible reingreso hospitalario, haciendo que el niño no tenga que pasar por el estrés de una hospitalización y disminuyendo así costes para el sector sanitario.

Así cómo también nos enseña el trabajo de Mucha, Selma e Silva (2015) los altos costos bien como los riesgos para los prematuros, nos señalan que invertir en atención primaria y prevención aún sigue siendo la mejor opción en este caso, indicando que el direccionamiento de los recursos deben de ser volcados para el ámbito de la salud pública con el objetivo de mejorar la asistencia a las mujeres embarazadas desde el momento inicial del embarazo hasta la Atención Temprana.

\section{BIBLIOGRAFÍA}

- Behrman, RE., Butler, AS., editors. (2007). Preterm birth: causes, consequences, and prevention. Washington, DC: The National Academies Press.

- Bonito, RR. (2012). Manual de Neonatología. México: Mc Graw Hill.

- Donabedian, A. (1986). La investigación sobre la calidad de la atención médica. Salud pública Méx. 28, 324-327.

- Hayakawa et al. (2010). Incidência de Reinternação de Prematuros com Muito Baixo Peso Nascidos em um Hospital Universitário. Esc Anna Nery Rev Enferm. 14 (2), 324-329.

- Howson, CP., Kinney, MV., Lawn, JE. (2012). Nacidos Demasiado Pronto: Informe de Acción Global sobre Nacimientos Prematuros. Nueva 


\section{Cultura de los Cuidados}

York: March of Dimes, PMNCH, Save the Children, Organización Mundial de la Salud.

- López, IC., García, MI. (2001) Enfermería Pediátrica. Manuales de Enfermería. 1. ${ }^{a}$ ed. Barcelona: Elsevier Masson.

- Manual de Descripción de los Grupos Relacionados por el Diagnósticos (AP-GRD v.25.0). (2010). Euskadi: Osakidetza.

- Mucha, F., Selma, CF., Silva, GAG. (2015). Frequência e características maternas e do recém nascido associadas à internação de neonatos em UTI no município de Joinville, Santa Catarina-2012. Rev. Bras. Saude Mater. Infant. 15(2).

- Oliván, JAS. Sistemas de información hospitalarios: el C.M.B.D. (1997). Scire. 3(2), 115-130.

- Paolillo, E., et al. Grupos Relacionados por el Diagnóstico (GRD). (2008). Experiencia con IRGRD en el Sanatorio Americano, sistema FEMI. Rev. Méd. Urug. 24(4), 257-265.
- Petrou, S. et al. (2003). The impact of preterm birth on hospital inpatient admissions and costs during the first five years of life. Pediatrics. 112, 1290-1297.

- Ramírez, FR., Hübner GME. (2002). Sobrevida, viabilidad y pronóstico del prematuro. Rev. méd. Chile. 130 (8), 931-938.

- Tamez, RN, Silva, MJP. (2002). Enfermería en la Unidad de Cuidados Intensivos Neonatal. Asistencia del recién nascido de alto riesgo. Rio de Janeiro: Guanabara Koogan.

- Tommiska, V., Tuomnien, R., Fellman, V. (2003). Economic costs of care in extremely low birthweight infants during the first two years of life. Pediatr Crit Care Med. 4, 157-163.

- Underwood, MA, Danielsen, B., Gilbert, WM. (2007). Cost, causes and rates of rehospitalization of preterm infants. Journal of Perinatology. 27, 614-619. 